\title{
marge: An API for Analysis of Motifs Using HOMER in $\mathrm{R}$
}

\author{
Robert A. Amezquita
}

2018-01-16

\section{Introduction}

Profiling of open chromatin regions through the assay for transposase-accessible chromatin (ATAC) and transcription factor binding via chromatin immunoprecipitation (ChIP) sequencing has increased our ability to resolve patterns of putative transcription factor binding sites at the genome-wide level. Popular tools such as HOMER and MEME have driven forward the analyses of sequence composition, deriving de novo motifs and searching for the enrichment of known motifs. However, their interfaces do not allow for the construction of parallel inquiries of multiple datasets. Furthermore, their results do not conform to formats amenable to 'tidy' analyses, presenting a significant bottleneck in motif analysis. Here, I present marge, a companion $R$ package that interfaces with HOMER to facilitate the construction of queries and to tidy results for further downstream analyses.

\section{Methods}

\section{Implementation}

marge is an $\mathrm{R}$ package that makes use of various tidyverse conventions, allowing for modern conventions of $\mathrm{R}$ programming and manipulations. marge interfaces directly with HOMER to construct queries, execute motif enrichment searches, and organize results. While this requires a separate installation of HOMER and introduces this long-term dependency, core HOMER routines have been stable for a long time, with infrequent updates. Given the popularity of HOMER, the syntax and construction of queries will thus be familiar to the audience utilizing the marge companion package. However, to conform to modern standards of software design, certain core routines, such as findMotifsGenome.pl which encompasses many different tasks, have been partitioned into distinct $\mathrm{R}$ functions. For example, motif analysis and motif location identification have been split up into distinct functions find_motifs_genome and find_motifs_instances, respectively.

\section{Operation}

marge is distributed on Bitbucket as an R package, and is compatible with Mac OS X, Windows, and Linux. The latest version of HOMER (v4.9 as of this writing) should be installed prior to marge, and configured with the desired genomes and feature sets. Subsequently, marge can be installed from the repository. $\mathrm{R}$ Package dependencies and system requirements are documented in the repository and automatically installed in conjunction with the marge package. Online documentation is also available at marge.aerobatic.io.

\section{Use Cases}

To demonstrate the functionality and utility of marge, we present a tutorial of core functionality and present potential use cases for high-throughput motif enrichment studies and subsequent downstream analyses. 


\section{Conventions}

Code objects shall be referred to in-line with the code font, whereas $\mathrm{R}$ functions will be referred to with a pair of parentheses following the function name, as in find_motifs_genome(). $\mathrm{R}$ code chunks will be presented as a block. Comments describing relevant portions of code are prepended by hashes (\#), and all else is runnable $\mathrm{R}$ code. Results of code chunks are printed in separate block below, with each line of output prepended by a double hash (\#\#). An example of code chunk and output formatting follows:

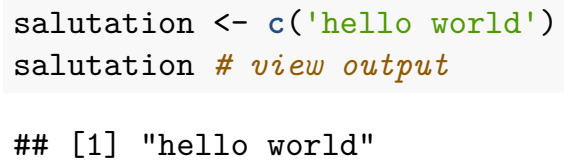

Lastly, documentation for functions can be accessed invoking through help utility in R. Online documentation with the same content is also available under the 'Reference' tab.

\section{Preamble}

The latest version of HOMER should be installed prior to installing marge as documented online in the introduction/install section. Following installation, HOMER packages should additionally be installed, which contains the necessary sequence data to perform motif enrichment, such as those that follow:

\#\# In a terminal/command-line

perl /path-to-homer/configureHomer.pl -install hg38 \# human sequence data

perl /path-to-homer/configureHomer.pl -install mm10 \# mouse sequence data

Additionally, users should take care to ensure that HOMER is added to the executable path, such that entering findMotifsGenome.pl on the command-line works as expected. For further details, we again refer to the introduction/install section of the HOMER documentation.

\section{Basic Usage}

What follows is a basic tutorial of a typical workflow in performing motif enrichment analysis from a given, single set of regions. This section will introduce the various verbs available in marge for this task.

\section{Check HOMER and Installed Packages}

To check that HOMER was installed properly and is detected by marge invoke the check_homer() function. Sequence annotation data for the genome of interest should also have been installed prior to running HOMER or marge. To check what is currently available via HOMER, simply run list_homer_packages (). For this analysis, either 'hg38' or 'mm10' packages can be installed, which include the human and mouse genomes and annotations, respectively.






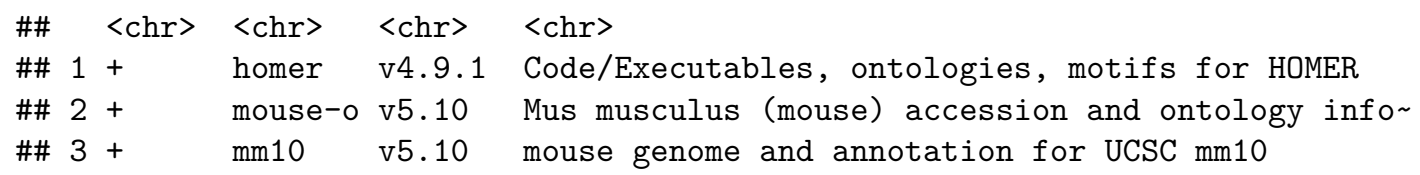

\section{Input data}

To begin, regions of interest should first be identified. For example, peak calls derived from ATAC-seq or ChIP-seq data are of particular interest for motif enrichment analyses. At minimum, such regions should contain three columns: chrom (chromosome), start, and end, describing the location of the region of interest. Additional columns are also allowed - suggested columns include a region identifier, gene annotations, distance to the gene, and properties such as expression/chromatin state.

A minimal set of regions is included with the marge package for testing, and can be loaded as follows:

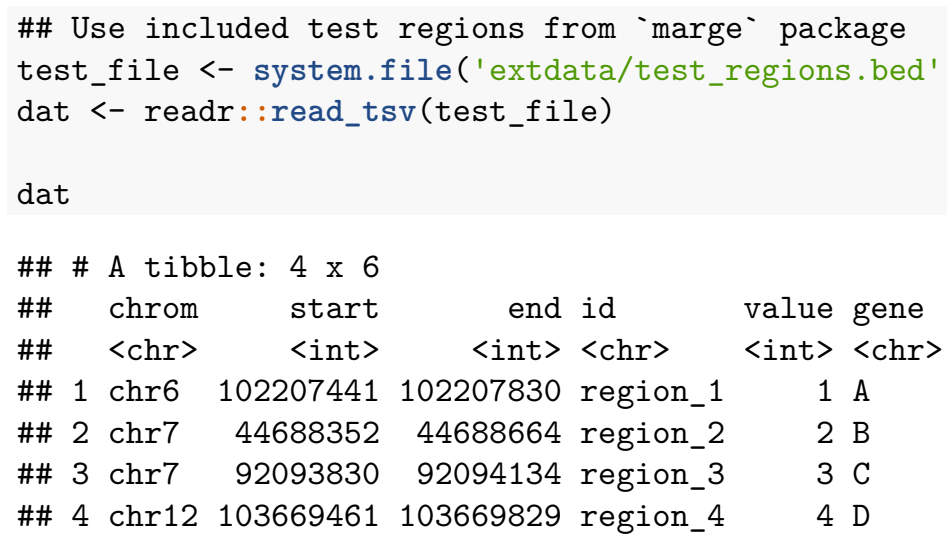

\section{Find Motifs Across the Genome}

From the regions of interest, enriched de novo and/or known motifs can be performed via the find_motifs_genome() function. This function connects to the HOMER utility findMotifsGenome.pl, and uses much of the same syntax, and so should be familiar to users of HOMER. The following code shows all available options, with documentation available online and via $\mathrm{R}$ for further details. Suffice it to say, a de novo and known motifs enrichment analysis is run on the test regions loaded prior, and writes results to the designated path directory (here, results_dir). Thus, find_motifs_genome() is run purely for its side-effect of creating a HOMER results directory.

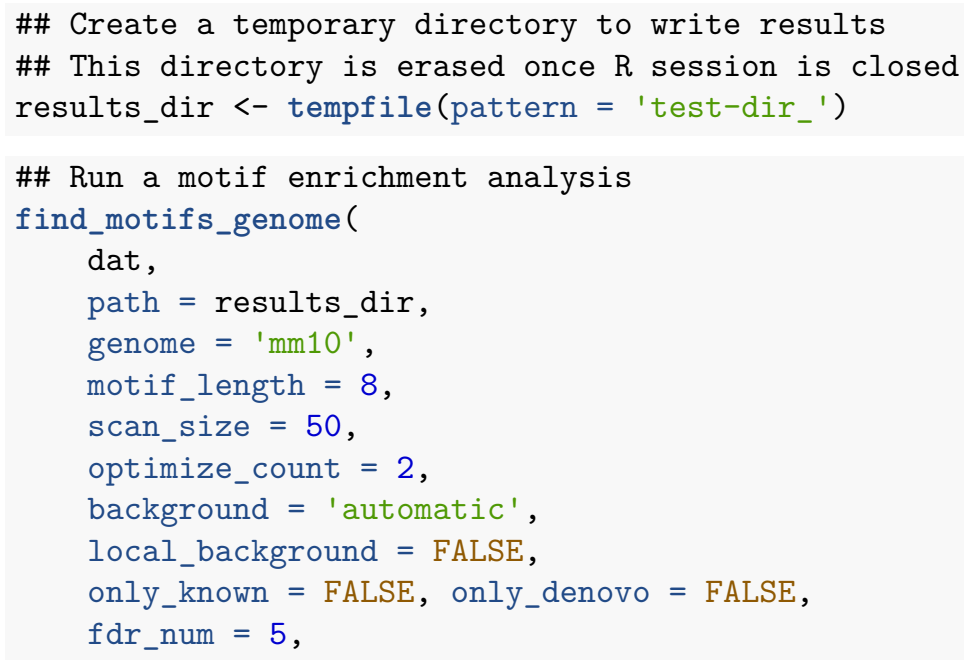




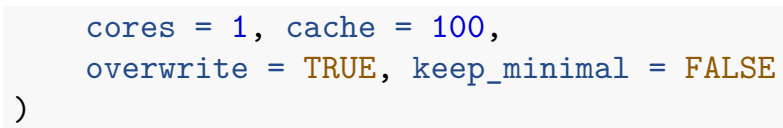

Note in particular the argument keep_minimal, which if set to TRUE, removes all extraneous HTML and logo images, retaining only the relevant enrichment results necessary for downstream analyses. If FALSE (the default), the output is exactly the same as if HOMER had been run from the command line with the given parameters.

\section{Load Denovo and Known Motif Enrichment Results}

HOMER produces two distinct files for de novo and known motif enrichments within the designated results directory, contained in homerMotifs.all.motif and knownResults.txt, respectively. These results can be read in using the respective read_*_results () functions, where $*$ is either denovo or known.

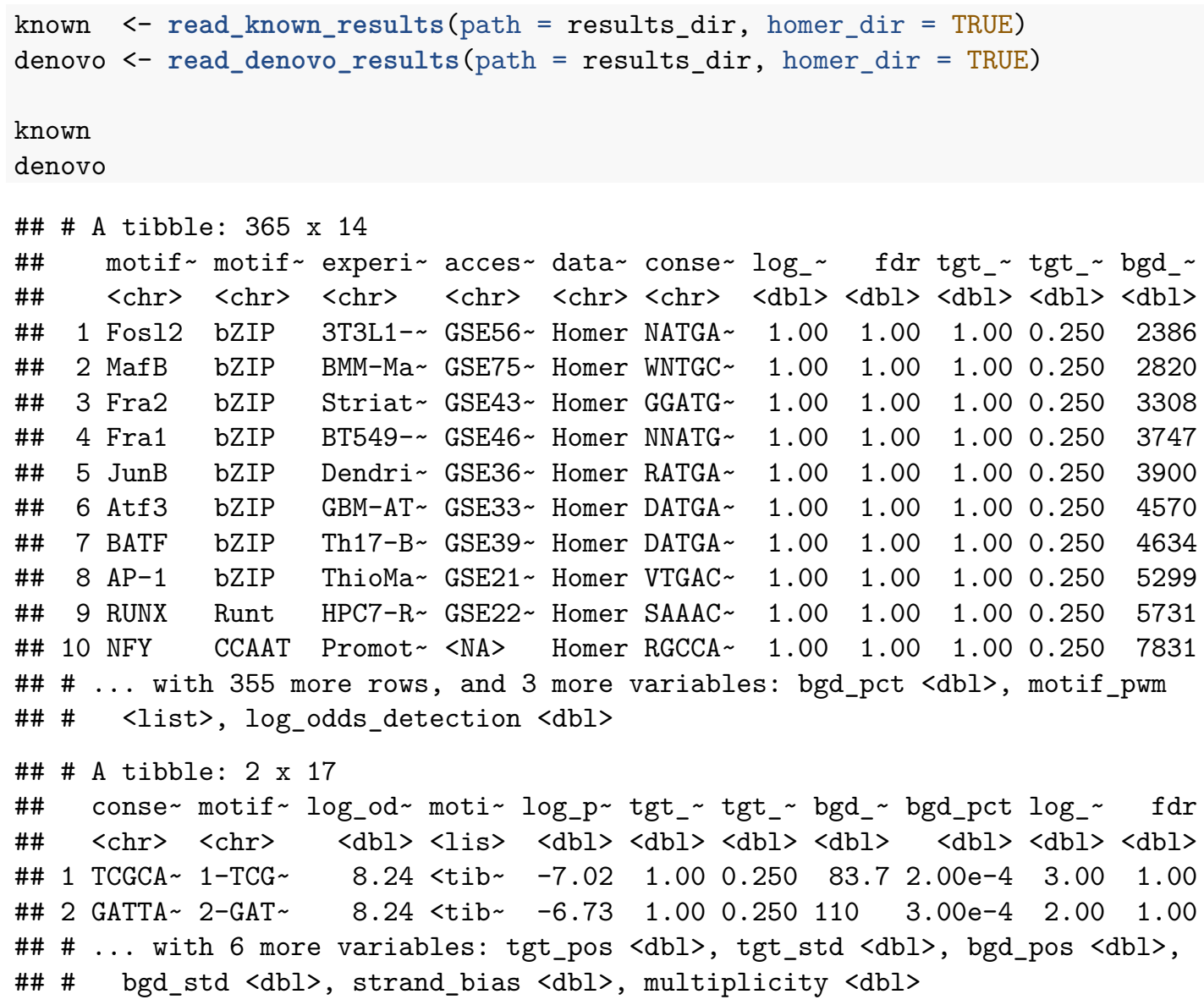

These functions specifically are responsible for the core routine which tidies the HOMER results, and can be pointed to any existing HOMER motif enrichment result files by setting homer_dir to FALSE. The output provides a tidied version of the default HOMER output, capturing the various characteristics of enrichments, such as the identity of the motifs, target and background number/percents of the motifs across the provided sequences, statistics of enrichment, and finally the associated motif position weight matrices (PWM) in the list-column motif_pwm. The columns are described further in the associated help files. 


\section{Accessing Motif PWMs}

One core task of interest is working with the identified de novo motif PWMs that were enriched across the regions of interest. To access a specific motif PWM, the following shows several ways of accessing them from the motif_pwm list-column in the known and denovo objects previously created.



Note the column names identifying the nucleotide, the rows for the ordered nucleotide position, and the frequencies at each cell, where each row sums to 1.

\section{Writing Motif PWMs - Manual}

To write a motif to a file, the helper function write_homer_motif () can be used, and values can be manually specified for motifs of interest.



\section{Writing Motif PWMs - Automatic}

Oftentimes, one wants to write all associated motif PWMs from a given de novo enrichment search. Given that write_homer_motif produces a single motif at a time, we need a way to produce a file for each row of the denovo object.

While there are various ways to iterate over the rows of the denovo object, such as with for-loops or the apply family of functions, below is shown a method using the purrr package from the tidyverse family, using the map() and walk() functions. Briefly, these functions apply a function to each element of a vector, 
thus allowing for iteration over the rows of an object such as those we created, known and denovo. map returns the transformed object, whereas walk calls the function for its side-effect, such as in the case of writing a file as in write_homer_motif(). Given that we need several varying inputs (all the arguments of write_homer_motif(), we use the variant pwalk() to allow for our many varying inputs. The resulting code below writes all of our denovo results (three motifs in total) to unique files in our temporary $\mathrm{R}$ results directory.

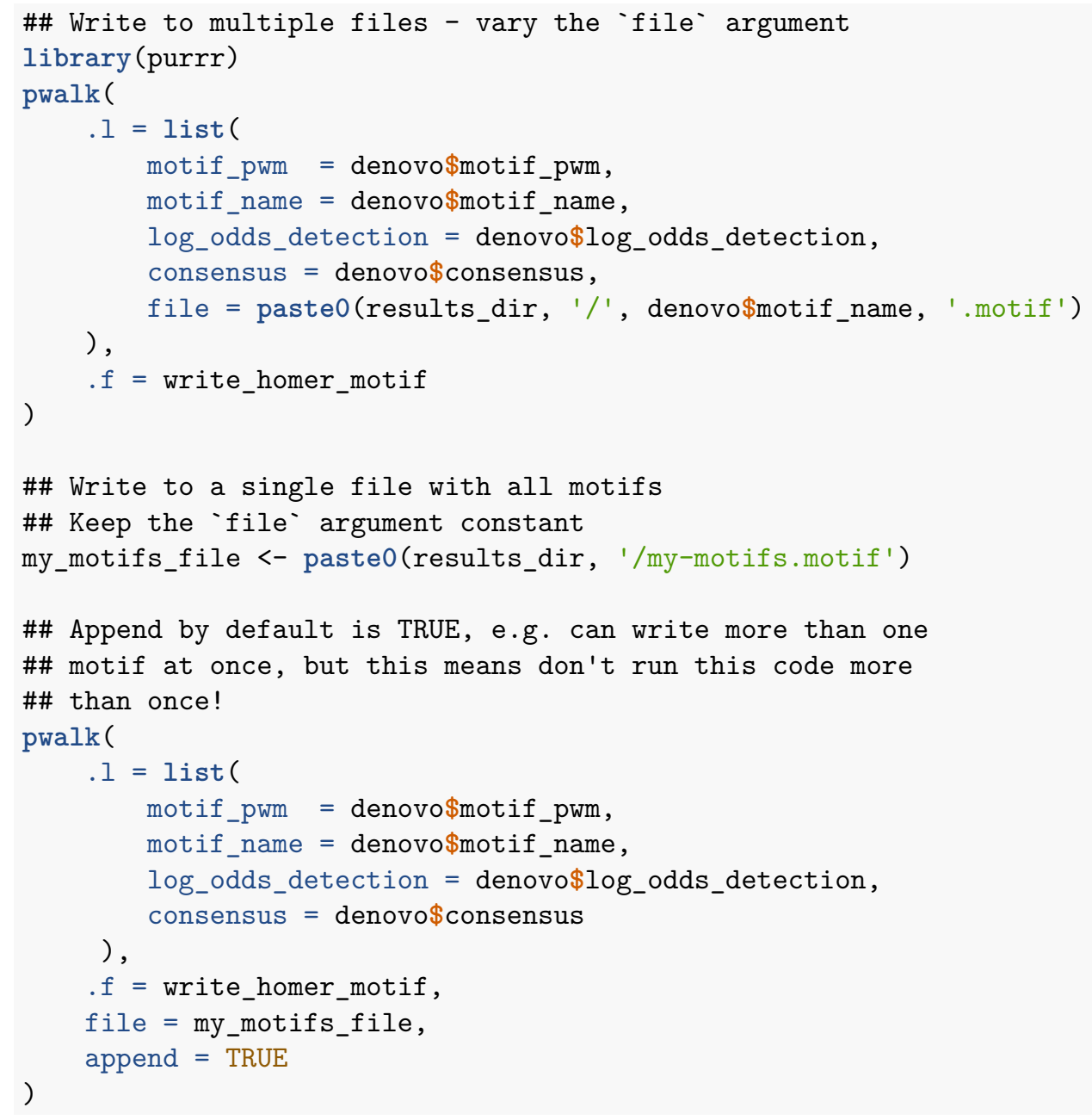

\section{Search for Instances of Motifs Across Regions}

Now that we have produced our motif PWM as a file, it can be used to map the motif back to where it occurs in our set of regions using the find_motifs_instances() function. While it is also a wrapper for findMotifsGenome.pl, it partitions out a unique piece of functionality that presents an entirely different type of results. Note that this function requires the use of a HOMER motif file that is written out (either by hand or by the write_homer_motif() function) as it contains the necessary parametrization for motif finding.

Also note that we can search for either a single motif or multiple motifs concurrently, where the multiple motifs are written into a single file, as shown above. Here, we will search for all three of the de novo motifs identified initially in our regions.

motif_instances_file <- paste0(results_dir, '/motif-instances.txt')

find_motifs_instances( 


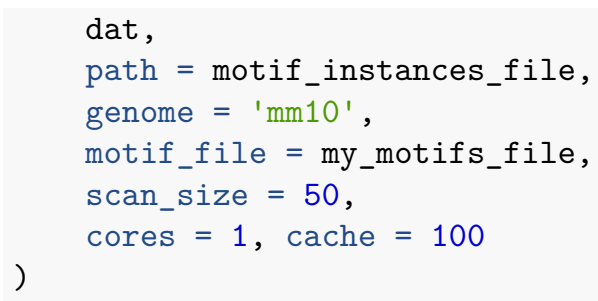

This function, similarly to find_motifs_genome() and write_homer_motif(), produces output that is written to into a file outside of $\mathrm{R}$, and similarly relies on a helper function to read the resulting output.

\section{Read Instances of Motifs Across Regions}

The function read_motifs_instances() works in tandem with find_motifs_instances() to read its output, returning the location and scores of the specified motifs from the previous step.

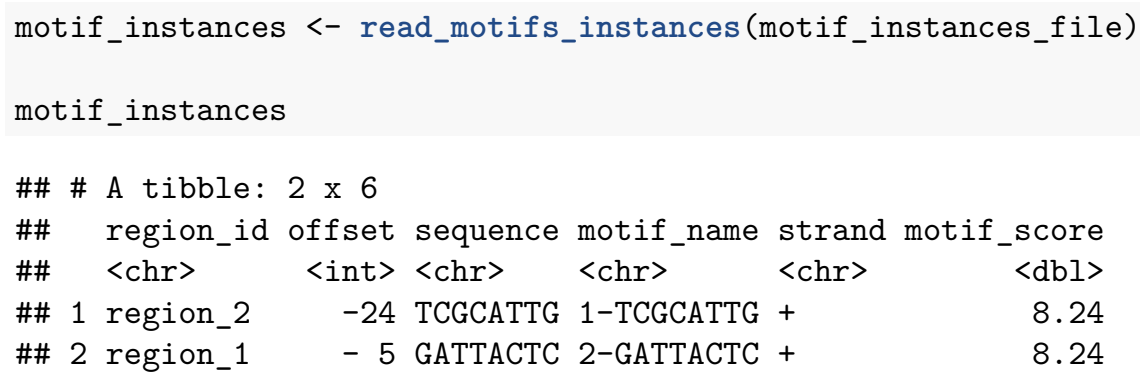

\section{Advanced Usage}

Using the verbs learned in the previous section, this part will focus on tying it all together to create a framework for testing for motif enrichment and subsequently analysing the results across multiple sets of regions in a tidy fashion. This section relies heavily on the tidyverse framework, and in particular will use the tools from purrr, $\operatorname{map}() /$ walk(), introduced in the previous section. Additionally, we will simulate a dataset using the valr package function bed_random(). While outside of the scope of this tutorial, valr provides additional functionality to work with genomic intervals using similar tidyverse conventions, and is recommended for manipulating regions of interest from high-throughput experiments.

Below are the libraries required for this next section.

\#\# Required libraries for advanced usage tutorial

library (valr)

library (dplyr)

library (tidyr)

library (purrr)

library (ggplot2)

\section{Constructing Multiple Simulated Sets of Regions}

By bringing the usage of HOMER into $\mathrm{R}$ via an API, it becomes possible to construct multiple queries, execute them all, and then read in the results from all the queries.

First, we will create a simulated set of intervals for which to test for motif enrichment, formatting the regions into a tibble object which allows for the creation of list-columns, which tidily encapsulate the genomic intervals. 


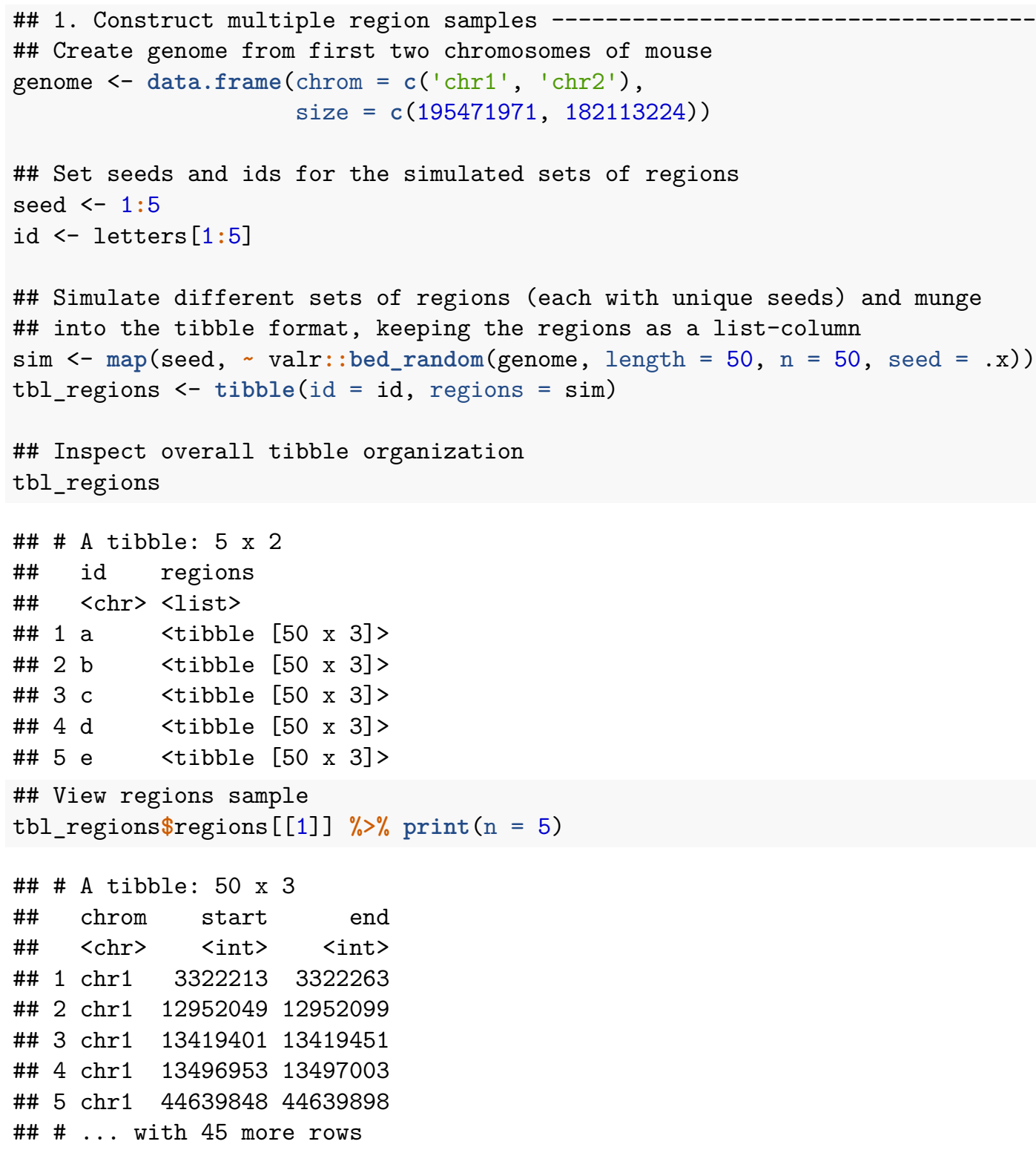

\section{Finding Motifs Across Multiple Sets of Regions}

The next step involves specifying the paths to the results directory for each respective set of regions. This is necessary for find_motifs_genome() to output the motif enrichment results for each set of regions separately (otherwise the results will be overwritten multiple times).

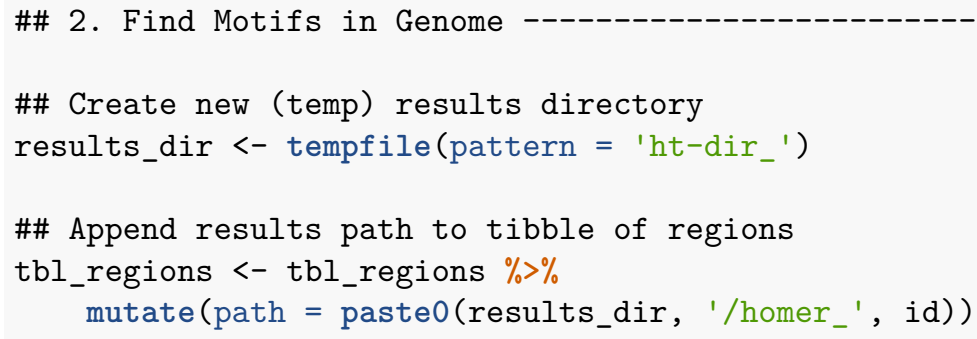






Now, using each set of regions and the unique paths for each set of regions, find_motifs_genome() can be run, varying across these two parameters, and setting as constant the various motif enrichment search parameters for all the different sets.

One other potential application not shown here is testing how these various parameters affect motif enrichment results if we were instead to keep the regions tested as constant instead. This is left as an exercise for the interested reader.



\section{Combining Results from Multiple Motif Enrichment Searches}

Following the motif enrichment search for each set of regions, the results can be read in, iterating over the variable path within the tbl_regions object to pull in the relevant results. The results are cleaned to remove the path and regions columns subsequently, and then expanded such that each individual motif enrichment result has its corresponding id as a separate variable.

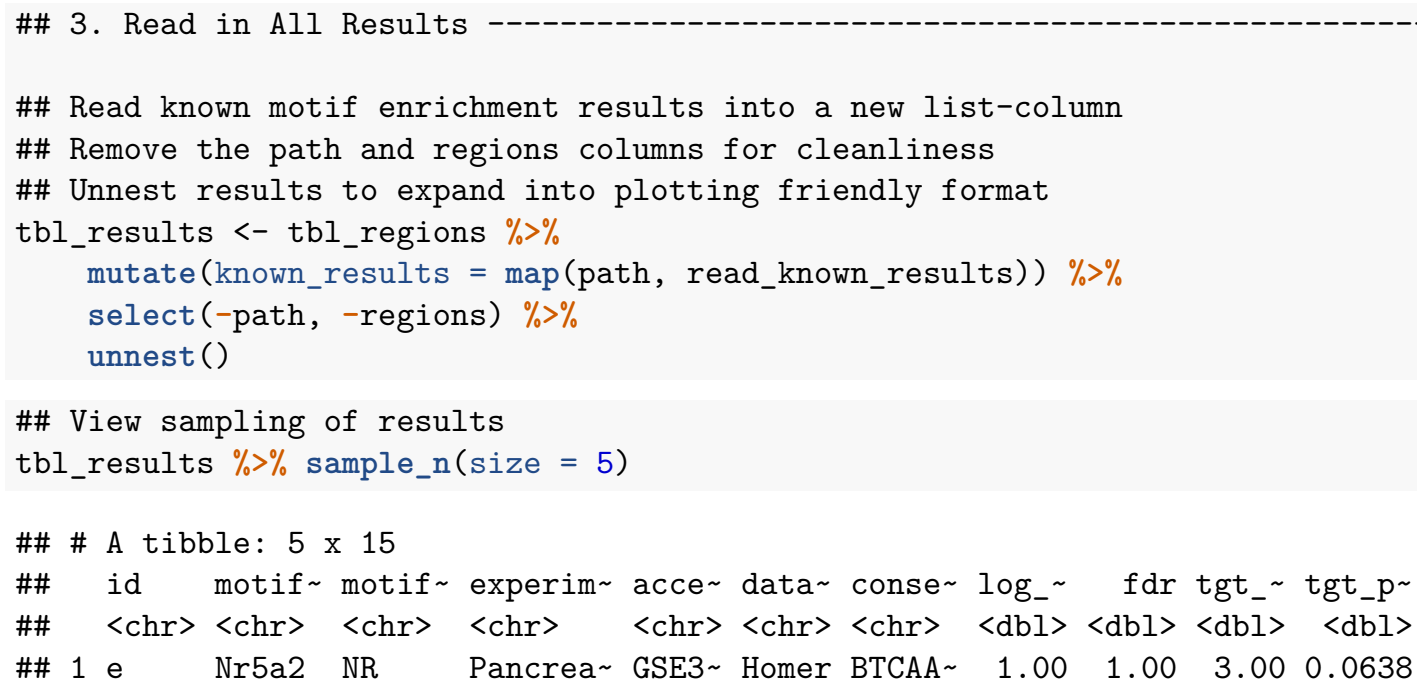




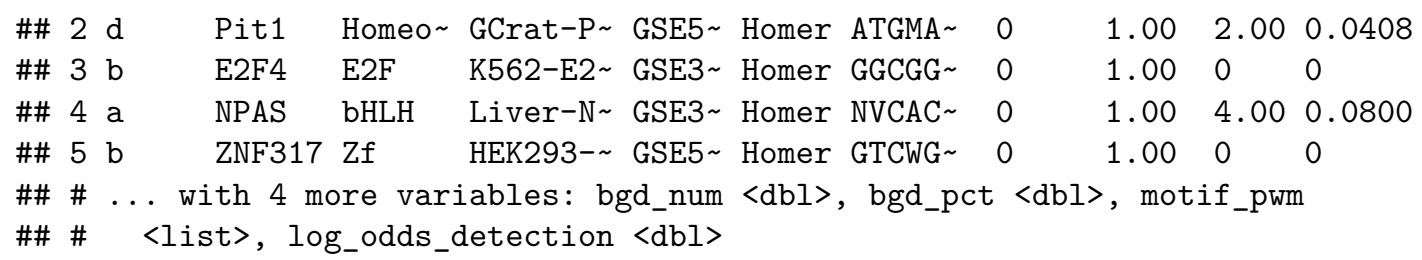

\section{Summarising Characteristics of Motif Enrichment Results}

In this tidy form, it becomes possible to summarise results on the basis of motif families, as opposed to simply individual motifs. Below, we use the id and motif_family columns to group the results, and summarise a given motif family by taking the most significant individual motif appearance as the summary statistic (e.g., the max - $\log 10 \mathrm{p}$-value).

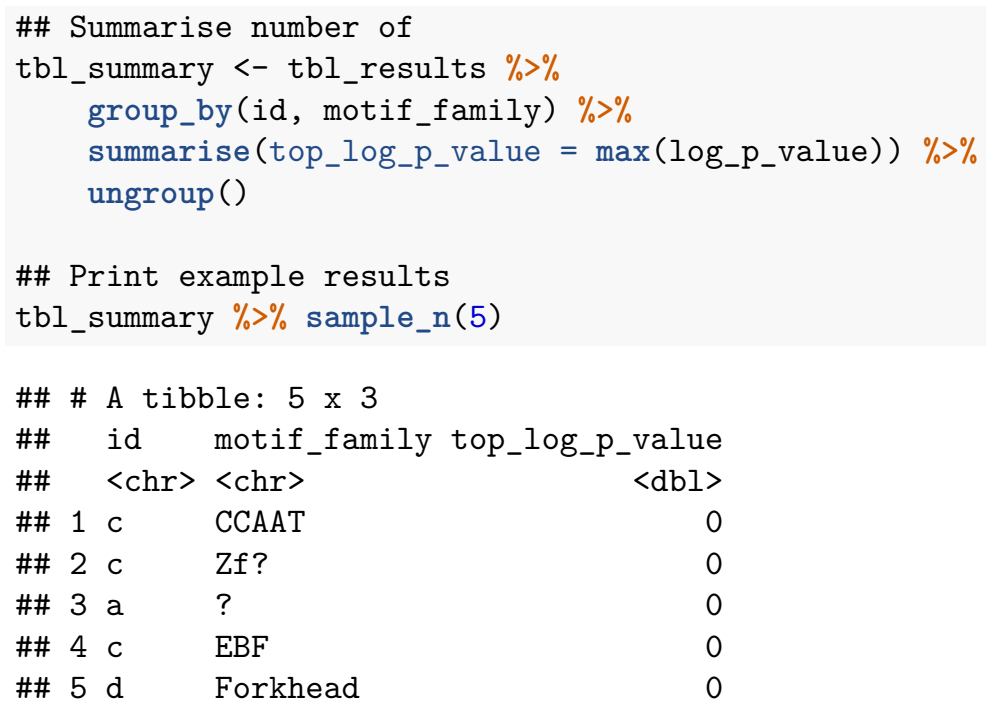

Note that few motifs appear significant in this simulated dataset, as regions were chosen at random, without a true biological correlate.

\section{Plotting Results}

Finally, we can inspect the results of our analyses using ggplot2 package conventions, given the tidiness of the results. Here we show a subset of the overall results for succinctness.

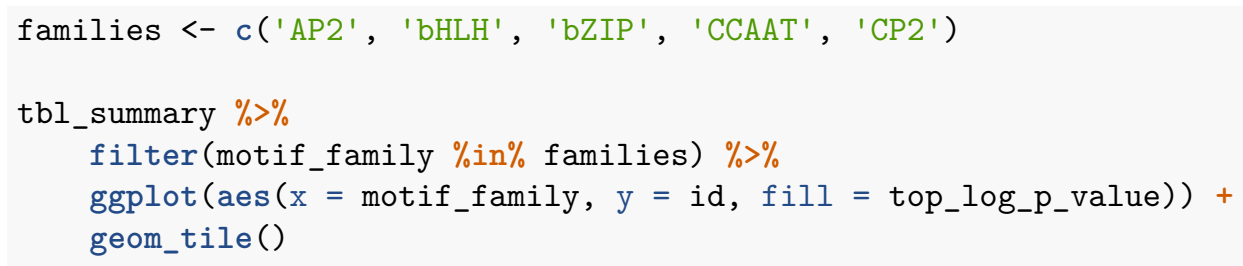




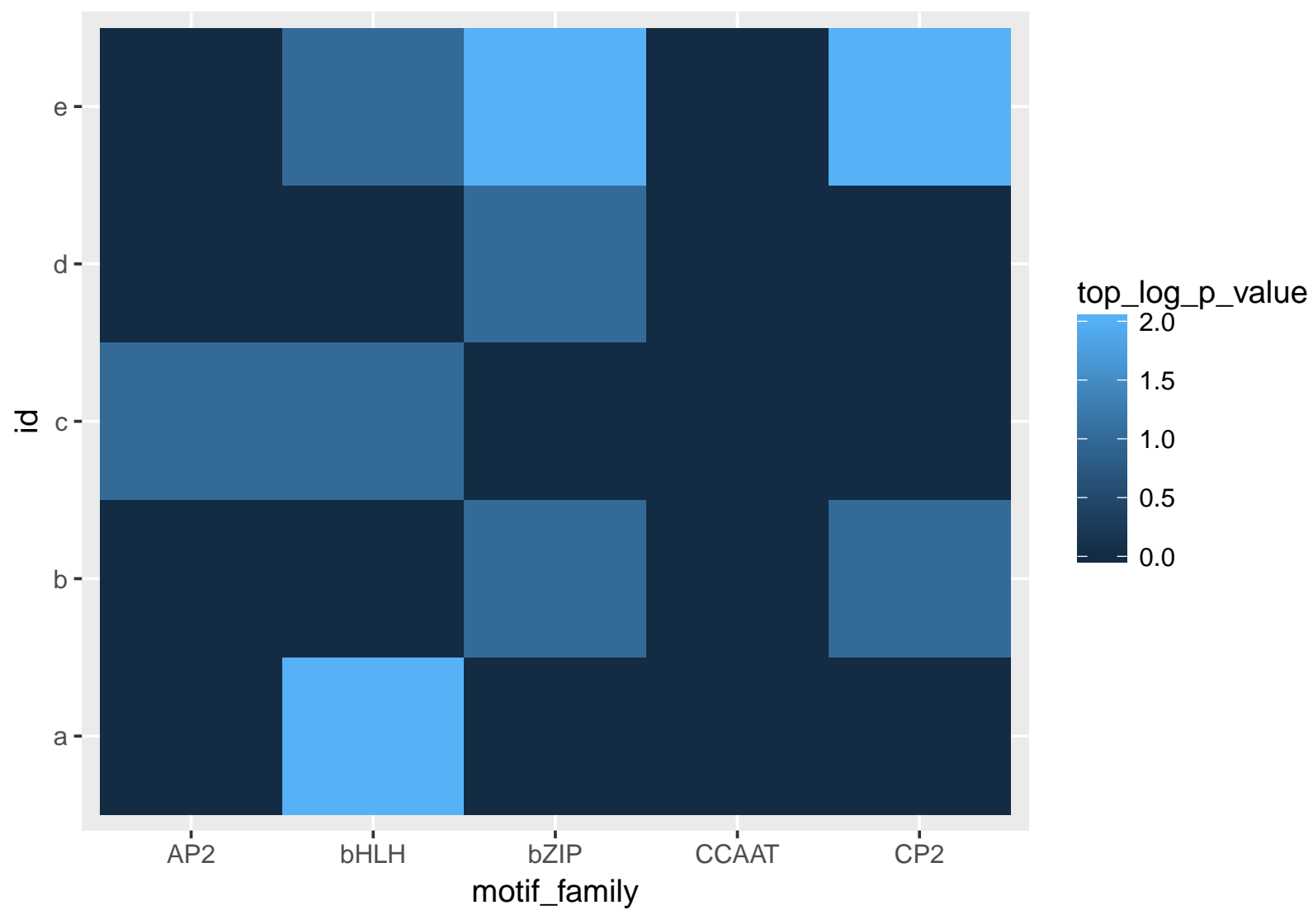

\section{Summary}

marge presents an $\mathrm{R}$ centric form of performing and analyzing motif enrichment results using the popular HOMER suite of tools, providing an easy-to-use interface and results in line with modern $\mathrm{R}$ idioms. We envision that marge will serve as a valuable tool to assist researchers in performing motif enrichment analyses quickly, easily, and reproducibly.

\section{Data and Software Availability}

Online documentation for HOMER and installation details can be found at: http://homer.ucsd.edu/homer/ marge can be installed via devtools using: devtools : install_bitbucket ('robert_amezquita/marge' , ref $=$ 'master')

The latest marge source code is available at: https://bitbucket.org/robert_amezquita/marge/

Stable versions of marge are located in the Downloads section of the source code repository, under the tab 'Tags', at: https://bitbucket.org/robert_amezquita/marge/downloads/

Online documentation for marge can be found at: https://marge.aerobatic.io/

\section{Competing Interests}

No competing interests were disclosed. 
bioRxiv preprint doi: https://doi.org/10.1101/249268; this version posted January 17,2018 . The copyright holder for this preprint (which was not certified by peer review) is the author/funder, who has granted bioRxiv a license to display the preprint in perpetuity. It is made available under aCC-BY 4.0 International license.

\section{Acknowledgements}

R.A.A. wrote the vignette, designed and implemented the software package. Jason Vander Heiden edited the manuscript and reviewed source code for clarity. Greg Finak contributed to code sussing out HOMER installation whereabouts. 